\title{
Three-dimensional infrared models of the interplanetary dust distribution
}

\author{
S. M. Kwon ${ }^{1}$ and S. S. Hong ${ }^{2}$ \\ ${ }^{1}$ Department of Science Education, Kangwon National University, Chunchon, Korea \\ ${ }^{2}$ Department of Astronomy, Seoul National University, Seoul, Korea \\ (Received December 8, 1997; Revised April 9, 1998; Accepted April 13, 1998)
}

\begin{abstract}
We have calculated the brightness of zodiacal emission by using the three dimensional optical models of zodiacal cloud. By comparing the calculated brightness distribution with the IRAS observations, we found that the cosine model is the best out of the three for describing the helioecliptic latitude dependence of dust distribution. We also found best parameters for the heliocentric variations of the dust temperature and volumetric absorption cross-section. Inclination and ascending node of the symmetry plane were deduced from annual variations of i) the peak offset latitude and ii) the pole brightness difference. Longitudes of the ascending node derived from i) and ii) are shown to be significantly different from each other.
\end{abstract}

\section{Introduction}

On the basis of visible zodiacal light (ZL), many models have been constructed for the three dimensional distribution of dust particles in the zodiacal cloud (Giese et al. (1986) and references therein). Although most models agree in that the density decreases by a factor of 2 within 0.2 to $0.3 \mathrm{AU}$ above the Earth orbit (Giese and Kneißel, 1989), the resulting morphology of the isodensity contours in the helioecliptic meridian looks quite different from model to model. Brightness distribution of the zodiacal emission (ZE) obtained by the IRAS (Hauser and Houck, 1986) may discriminate the models from each other.

\section{Model Calculations}

\subsection{IR brightness integral}

To obtain the ZE brightness at wavelength $\lambda$, we numerically calculate the following IR brightness integral:

$$
Z\left(\Lambda-\Lambda_{\odot}, \beta ; \lambda\right)=\int_{0}^{\infty} n\left(r, \beta^{\prime}\right) \sigma_{\mathrm{abs}}(r, \lambda) B_{\lambda}[T(r)] d l,
$$

where $n\left(r, \beta^{\prime}\right)$ denotes the dust number density at position $\left(r, \beta^{\prime}\right), \sigma_{\mathrm{abs}}(r, \lambda)$ is the mean absorption cross-section of the dust particles, and $B_{\lambda}[T(r)]$ means the Planck function evaluated with average temperature $T(r)$ of the particles at distance $r$ from the Sun. As shown in Fig. 1, the heliocentric latitude $\beta^{\prime}$ is measured from the symmetry plane. By calling $\beta^{\prime}$ the heliocentric symmetry-plane latitude we distinguish it from the heliocentric ecliptic latitude $\beta_{\circ}$ and from the usual geocentric ecliptic latitude $\beta$. Inclination angle of the symmetry plane is denoted by $i$, the angle $\eta$ orients the line of nodes of the plane with respect to the Sun-Earth direction, and $\phi$ is the heliocentric longitude of $\left(r, \beta^{\prime}\right)$ measured from the same direction. These angles satisfy the relation $\sin \beta^{\prime}=\cos \beta_{\circ}-\sin i \cos \beta_{\circ} \sin (\eta+\phi)$.

Copy right (c) The Society of Geomagnetism and Earth, Planetary and Space Sciences (SGEPSS); The Seismological Society of Japan; The Volcanological Society of Japan; The Geodetic Society of Japan; The Japanese Society for Planetary Sciences.
In most analyses of the ZL observations an expression of the form $n(r) f\left(\beta^{\prime}\right)$ is used to portray the three dimensional distribution of the dust particles. The function $n(r)$ is for heliocentric distribution, here $r$ being measured in the symmetry or the ecliptic plane; while $f\left(\beta^{\prime}\right)$ describes distribution over the heliocentric symmetry-plane latitude. As an approximation for $n(r)$ one often adopts a power law relation of the form

$$
n(r)=n_{\circ}\left(\frac{r_{\circ}}{r}\right)^{v},
$$

where $n_{\circ}$ is the number density at a reference distance $r_{\circ}$, for which $1 \mathrm{AU}$ is usually taken (cf. Leinert et al., 1981). A variety of models have been proposed for $f\left(\beta^{\prime}\right)$. Among them are the ellipsoid model $f\left(\beta^{\prime}\right)=\left[1+\left(6.5 \sin \beta^{\prime}\right)^{2}\right]^{-0.65}$ by Giese and Dziembowski (1969), the fan model $f\left(\beta^{\prime}\right)=$ $\exp \left[-2.1\left|\sin \beta^{\prime}\right|\right]$ by Leinert et al. (1981), and the cosine model $f\left(\beta^{\prime}\right)=0.15+0.85 \cos ^{28} \beta^{\prime}$ by Rittich (1986).

The model parameters were fixed at the values suggested by the authors of each model and were not changed during the calculations of ZE. By doing so, we could check compatibility of the optical 3-dimensional models with the IRAS observations of zodiacal emission. Hong and Kwon (1991) showed that the ellipsoid model is in an accordance with the Gegenschein part of the visible ZL. In analyses of the infrared ZE, however, many investigators (Hauser et al., 1985; Murdock and Price, 1985; Deul and Wolstencroft, 1988; and Rowan-Robinson et al., 1990) preferred fan-type models. It is then interesting to see which of the optical models is in agreement with the ZE observations.

We let a power law of index $\delta$

$$
T(r)=T_{\circ}\left(\frac{r_{\circ}}{r}\right)^{\delta}
$$

describe the variation of dust temperature with $r$. Here, $T_{\circ}$ denotes the dust mean temperature at the Earth orbit. Since it is hard to de-couple radial dependence of the dust number density from that of the absorption cross-section, we simply assign to the volumetric absorption cross-section $n(r) \sigma_{\mathrm{abs}}(r, \lambda)$ 


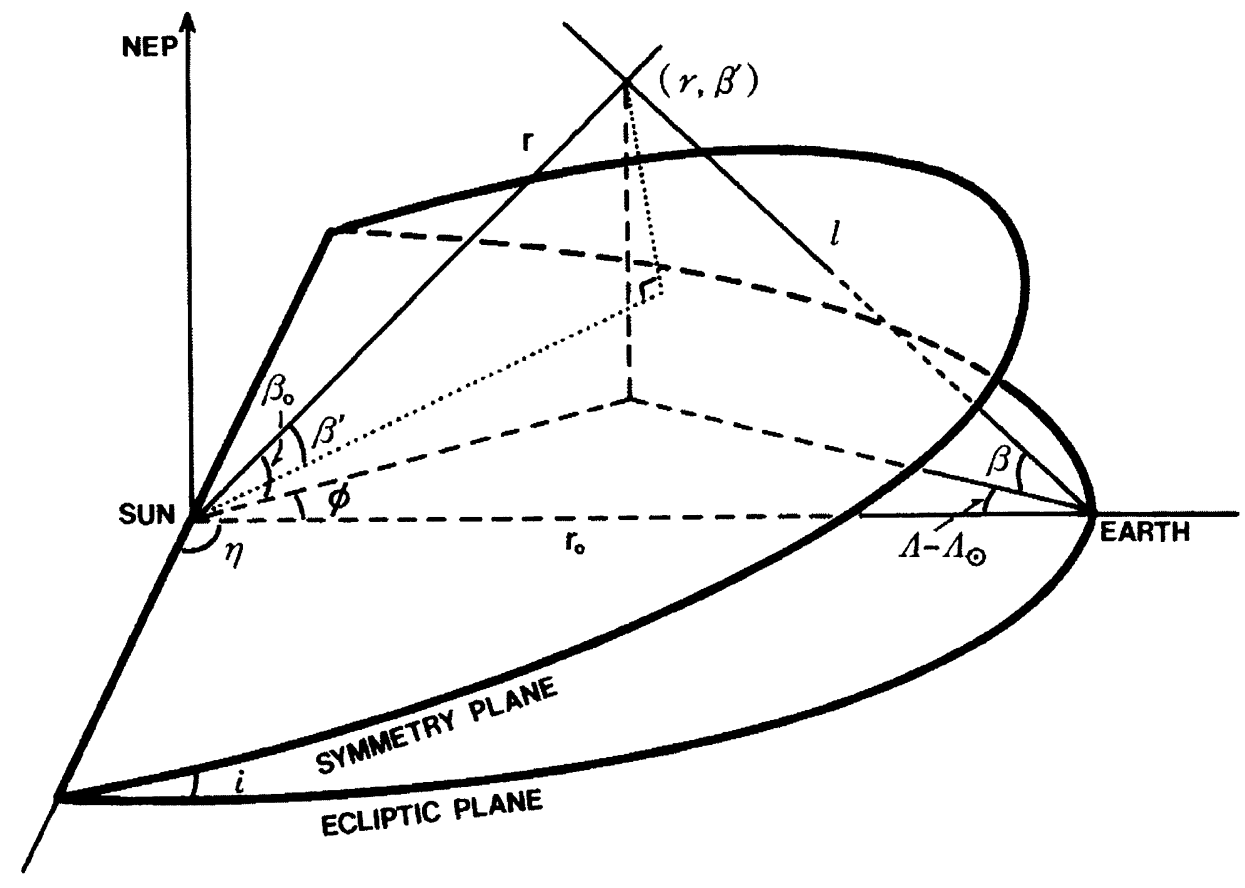

Fig. 1. Geometry involved in the IR brightness integral.

a power law relation of another index:

$$
\zeta(r ; \lambda) \equiv n(r) \sigma_{\mathrm{abs}}(r, \lambda)=\zeta_{\circ}(\lambda)\left(\frac{r_{\circ}}{r}\right)^{\gamma},
$$

where $\zeta_{\circ}(\lambda)$ denotes its value at the earth orbit.

\subsection{IR brightness distribution in the ecliptic plane}

If optical properties of various dust species and their mixing ratios are uniform over interplanetay space and the total number density follows a single power law relation, then the indices $\gamma, v$ and $\delta$ all become constants of $r$. However, many studies (for example, Hong and Um (1987), Hong and Kwon (1988), Temi et al. (1989), and Levasseur-Regourd and Dumont (1990)) indicate that power law relations with fixed indices are inadequate for the $\mathrm{ZE}$ observations. In this study we will have a flexibility of varying both $\gamma$ and $\delta$ with heliocentric distance. For the volumetric absorption crosssection we take

$$
\gamma(r)=\gamma_{\circ}-\frac{\Delta \gamma}{\pi} \tan ^{-1}\left(\frac{r-r_{\circ}}{\mathrm{AU}}\right)
$$

with $\gamma_{\circ}=1.0$ and for the dust mean temperature with $\delta_{\circ}=$ 0.4

$$
\delta(r)=\delta_{\circ}+\frac{\Delta \delta}{\pi} \tan ^{-1}\left(\frac{r-r_{\circ}}{\mathrm{AU}}\right) .
$$

The brightness distributions of the ZE at 12 and $25 \mu \mathrm{m}$ were calculated by integrating Eq. (1), with Eqs. (5) and (6) being substituted for $\gamma$ and $\delta$, respectively. By comparing the calculated distributions in the ecliptic plane with the IRAS observations, one may determine the best values for the parameters $\Delta \gamma, \Delta \delta$, and two $\zeta_{\circ}$ 's.

There are calibration differences among the different versions of the Zodiacal Observations History File (ZOHF) (Vrtilek and Hauser, 1995). In the comparison done in Fig. 2 we have used the ZOHF release 2, which was also used

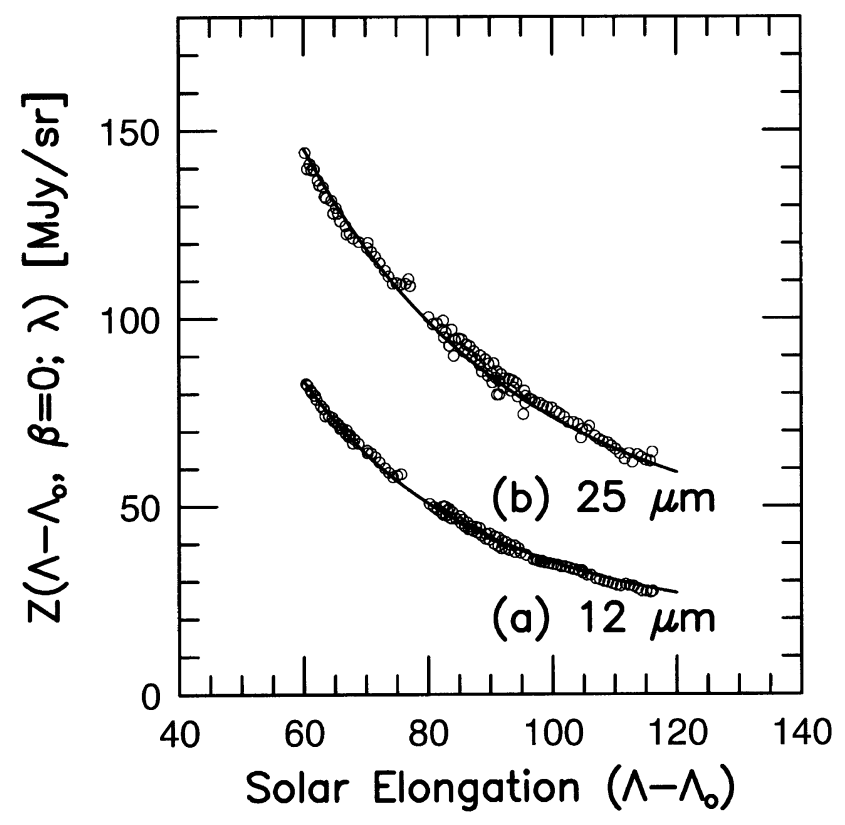

Fig. 2. Distribution of the IR brightness with differential solar elongation. The IRAS data (open circles) at (a) $12 \mu \mathrm{m}$ and (b) $25 \mu \mathrm{m}$ are compared with the model calculations (solid lines).

by Reach (1991). The best parameter values are $\Delta \gamma=$ $0.6, \Delta \delta=0.3$, and $\zeta_{\circ}(12 \mu \mathrm{m})=9.7 \times 10^{-21} \mathrm{~cm}^{-1}$ and $\zeta_{\circ}(25 \mu \mathrm{m})=1.3 \times 10^{-20} \mathrm{~cm}^{-1}$. The calibration difference may change the $\zeta_{\circ}$-values, but its effects on $\Delta \delta$ and $\Delta \gamma$ are completely negligible. Heliocentric variations of the resulting $T(r)$ (dots) and $\zeta(r)$ (solid line) at $12 \mu \mathrm{m}$ are compared in Fig. 3 to the ones with fixed indices (dashed lines). The dust temperature varies with logarithmic gradient shallower 


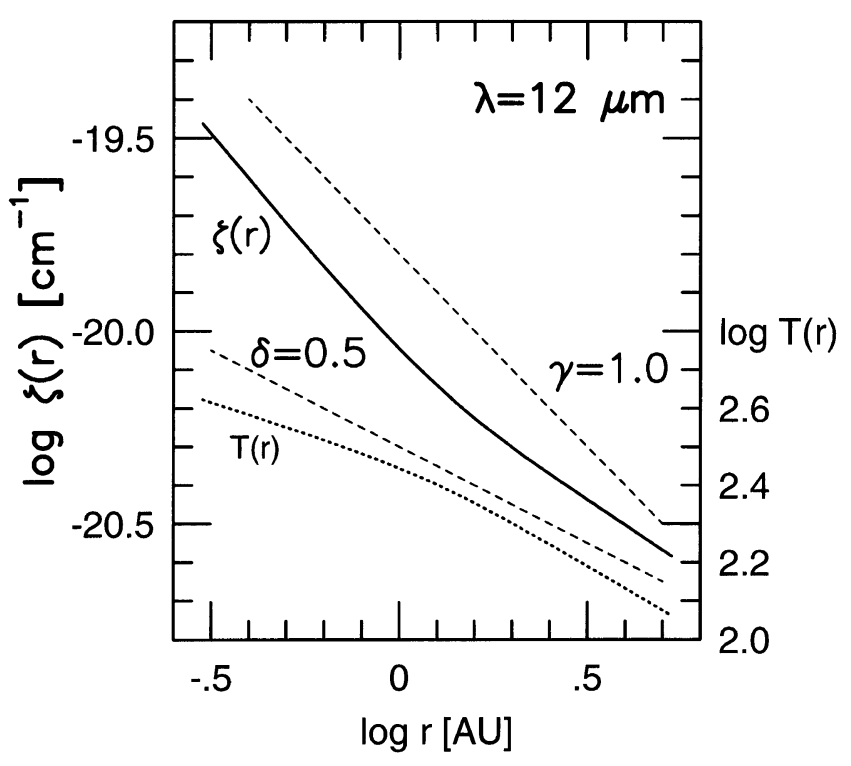

Fig. 3. Heliocentric variations of $\zeta(r)$ at $12 \mu \mathrm{m}$ and $T(r)$ are shown for the best-fit indices of $\gamma(r)$ and $\delta(r)$. Single power-law relations with $\gamma=1.0$ and $\delta=0.5$ are shown by dashed lines for comparison.

than 0.5 up to $3 \mathrm{AU}$, and the gradient slowly reaches its limiting value 0.55 at $r \simeq 8 \mathrm{AU}$. The volumetric absorption cross-section $\zeta(r)$ varies more steeply within the Earth orbit than beyond it.

\subsection{IR brightness distribution off the ecliptic plane}

We are now to find out best models for the distribution of density over heliocentric symmetry-plane latitude. For each of the three optical models, we integrated Eq. (1) with $\Lambda-\Lambda_{\odot}$ fixed at $90^{\circ}$ and $\beta$ varying from $-90^{\circ}$ to $90^{\circ}$. Details of the brightness profile over $\beta$ depend on the orientation of the symmetry plane, which enables us to locate the symmetry plane in terms of $i$ and $\Omega$.

The calculated brightness profiles over the helioecliptic meridian are directly compared, in Fig. 4, with the corresponding IRAS (thick solid lines) observations at $12 \mu \mathrm{m}$ and $25 \mu \mathrm{m}$. In the figure the dotted, solid, and dashed lines are for the cosine, fan, and ellipsoid models, respectively. Out of the three the cosine model delivers, in an overall sense, the best agreement with the IRAS observations.

The brightness profiles from the fan model show sharp peaks near the symmetry plane and broad wings towards the ecliptic poles. And the pole brightness at $25 \mu \mathrm{m}$ shows an excess of $\sim 7 \mathrm{MJy} / \mathrm{sr}$ over the observed value. The profiles from the ellipsoid model do not have such peaks near the symmetry plane, and are very similar to the ones from the cosine model in the range $-20^{\circ} \lesssim \beta \lesssim 20^{\circ}$. However, it also shows a significant excess of brightness towards the poles.

\section{Symmetry Surface}

It has been known that the surface of maximum dust density does not coincide with the ecliptic plane. We usually call the maximum density surface the symmetry plane, under the notion that it would form a plane. If it is a plane, one set of $i$ and $\Omega$ uniquely locates the surface with respect to the ecliptic plane. Many investigators deduced the $i$ and $\Omega$ sets from the ZL and ZE observations. (See the references in Ta- ble 1 and some recent discussions by Ishiguro et al. (1996) and Dermott et al. (1997).) As can be seen from Table 1, the results depend on the data and the deduction method. This conflicting situation was interpreted as an indication of warped nature for the maximum density surface (Misconi, 1980; Vrtilek and Hauser, 1995). We also assume a planar surface, not because the maximum density surface is planar, but because an accumulation of $i$ and $\Omega$ sets would eventually help us define its nature.

The ZL or ZE profile over $\beta$, at a fixed elongation say $90^{\circ}$, shows its maximum not necessarily at $\beta=0$. The peak occurs slightly off the ecliptic plane. If the maximum density surface forms a plane, the offset amount would vary sinusoidally with time of the year. A monitoring of the peak latitude $\beta_{\text {peak }}$ over a year would determine $i$ and $\Omega$. The difference in brightness between the north and south ecliptic poles would also show a sinusoidal variation with time. The IRAS has in fact done such monitorings.

We have numerically generated a series of ZE profiles over $\beta$ and determined $\beta_{\text {peak }}$ as a function of $\eta$. As far as the peak brightness latitude is concerned, the $f\left(\beta^{\prime}\right)$ models are of no consequences. By comparing the resulting run of $\beta_{\text {peak }}$ with the $12 \mu \mathrm{m}$ data of ZOHF release 2, we were able to fix $i$ and $\Omega$. The best-fit shown in Fig. 5 was made by $i=1 .{ }^{\circ} 5 \pm 0 .^{\circ} 1$ and $\Omega=52^{\circ} \pm 2^{\circ}$ for the trailing scan (filled circles), and for the leading scan (open circles) by $i=1 .{ }^{\circ} \pm \pm 0 .^{\circ} 1$ and $\Omega=59^{\circ} \pm 2^{\circ}$. The trailing and leading scans could not be fitted by a single set of $i$ and $\Omega$. By using the ZOHF release 3 , Vrtilek and Hauser (1995) obtained $i\left(=1 .^{\circ} 54\right)$ same as ours, but their $\Omega=40 .^{\circ} 9$ is different from our value $52^{\circ}$ to $59^{\circ}$. The discrepancy is due to the difference in comaprison data; our result based on the ZOHF release 3 agrees with theirs.

A run of the pole brightness difference was also made from the generated profiles, and compared with the IRAS observations of ZHOF release 2. The solid, dashed, and dotted lines in Fig. 6 are from the cosine, ellipsoid, and fan models, respectively. Equally good fits were made by the cosine and the ellipsoid models with the same set of $i=$ $2 .^{\circ} 3 \pm 0 .^{\circ} 1$ and $\Omega=75^{\circ} \pm 2^{\circ}$. As can be seen from the figure, the cosine and ellipsoid models are hardly distinguishable from each other. However, the fan model differs from the other two. The comparison done with the ZOHF release 3 gave us the same $\Omega=75^{\circ} \pm 2^{\circ}$, but the inclination value was reduced to $i=1 .{ }^{\circ} 9 \pm 0 .^{\circ} 1$. Using the same ZOHF release 3 , Vrtilek and Hauser (1995) placed the ascending node at $\Omega=$ $76 .^{\circ} 1$, but with the method based on geometrical fitting they could not determine the inclination value.

Phase of the annual variation curve for the pole brightness difference is solely determined by $\Omega$; while its amplitude is controlled by the column densities along the two directions of ecliptic poles. Therefore, the amplitude should depend on the model of $f\left(\beta^{\prime}\right)$ and the inclination as well. For a given model of $f\left(\beta^{\prime}\right)$, the larger the observed amplitude is, the higher the derived $i$ should be. This enables us to discriminate the three dimensional models of density from each other. This is also why the two sets of ZOHF yield different values for the inclination.

The visible $\mathrm{ZL}$ renders $i=2^{\circ}$ to $3^{\circ}$ and $\Omega=87^{\circ}$ to $100^{\circ}$, which roughly agree with the IR results from the pole 


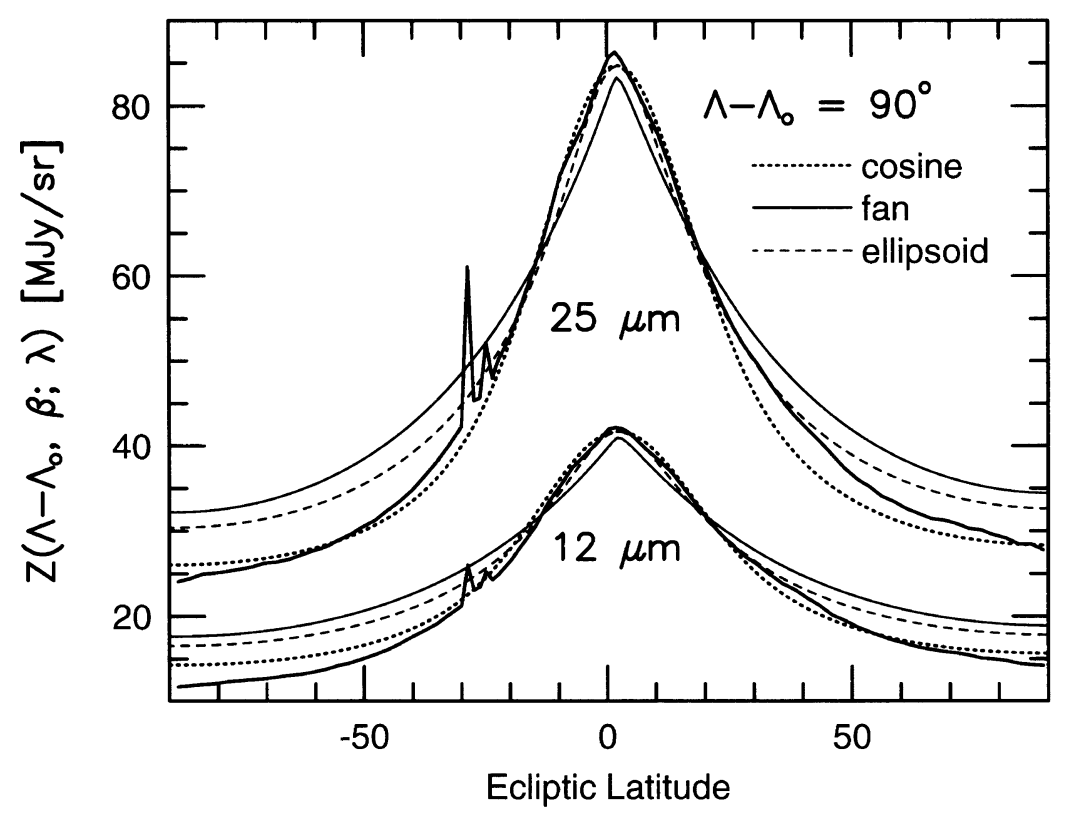

Fig. 4. The ecliptic latitude profiles (thick solid lines) of the IRAS brightness observed at (a) $12 \mu \mathrm{m}$ and (b) $25 \mu \mathrm{m}$ are compared with the results (dotted lines) from the best-fit cosine model. The results from the fan (thin solid lines) and the ellipsoid (dashed lines) models are also given for comparison.

Table 1 . Various $i$ and $\Omega$ values for the symmetry plane.

\begin{tabular}{lllll}
\hline Wavelength & Method & $i\left({ }^{\circ}\right)$ & $\Omega\left({ }^{\circ}\right)$ & References \\
\hline Visible & Helios 1 and 2 & 3.0 & 87 & Leinert et al. (1981) \\
& Gegenschein model & $2.0 \pm 0.5$ & $100 \pm 20$ & Hong and Kwon (1991) \\
\hline IR & Latitude of peak brightness & 1.5 & 55 & Hauser et al. (1985) \\
& $1.47 \pm 0.10$ & $50 \pm 4$ & Dermott et al. (1986) \\
& $1.30 \pm 0.05$ & $45.5 \pm 1.0$ & Deul and Wolstencroft (1988) \\
& $1.45 \pm 0.1$ & $53 \pm 1$ & Reach (1991) \\
& $1.54 \pm 0.01$ & $40.9 \pm 0.4$ & Vrtilek and Hauser (1995) \\
& & $1.5 \pm 0.1$ & $(52-59) \pm 2$ & This work \\
& $1.5 \pm 0.1$ & $(40-45) \pm 2$ & This work \\
\hline IR & 2.5 & 78 & IRAS Expl. Suppl. \\
& Pole brightness difference & $2.24 \pm 0.02$ & $70.0 \pm 1.0$ & Deul and Wolstencroft (1988) \\
& $\ldots$ & $76.1 \pm 0.3$ & Vrtilek and Hauser (1995) \\
& & $2.3 \pm 0.1$ & $75 \pm 2$ & This work \\
& $1.9 \pm 0.1$ & $75 \pm 2$ & This work ${ }^{a}$ \\
\hline
\end{tabular}

${ }^{a}$ Obtained from the release 3.0 of ZOHF.

brightness diffference. But the two set of results from the peak offset and the pole brightness difference do not agree with each other, particularly for the longitude of ascending node.

\section{Conclusion and Discussion}

We have seen that the change of mean dust temperature with heliocentric distance $r$ can not be described by a power law of single exponent. The raidal change in the exponent $\delta(r)$ as given in Eq. (6) suggests that mean dust properties vary systematically with distance from the sun. If multispecies nature is accepted for the zodiacal dust particles (Hong and Um, 1987; Hong and Kwon, 1988; LevasseurRegourd and Dumont, 1990; Kneißel and Mann, 1991; Reach, 1991), this kind of systematic variation can be an indication of changing mixture ratios.

We have shown that the fan model from ZL studies is incompatible with the IRAS observations of ZE. Reasonably 


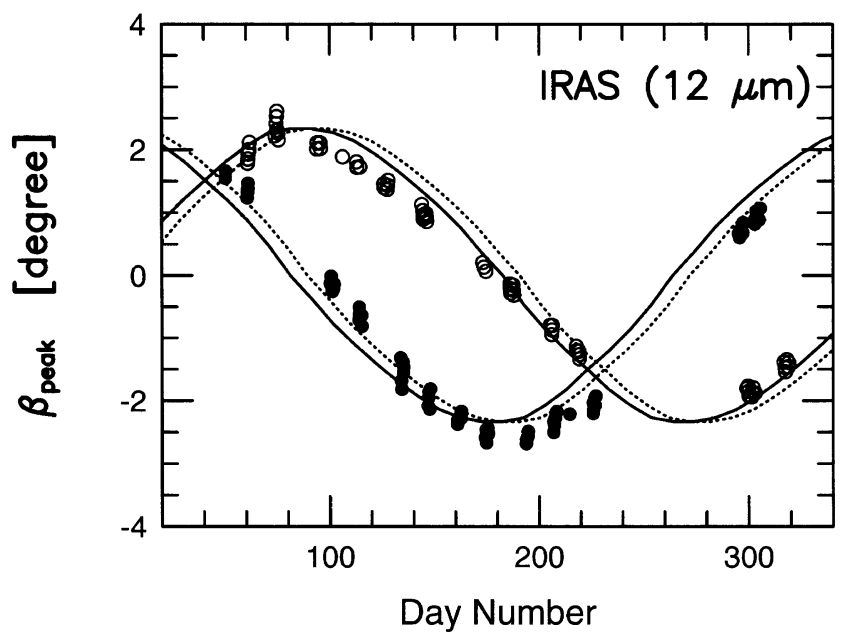

Fig. 5. The observed annual variation of the peak brightness latitude is compared with that from the model calculation. The leading scans (open circles) at $12 \mu \mathrm{m}$ are fitted with $i=1 .^{\circ} 5$ and $\Omega=59^{\circ}$ (solid line); while the trailing ones (filled circles) are with $i=1^{\circ} .5$ and $\Omega=52^{\circ}$ (dotted line).

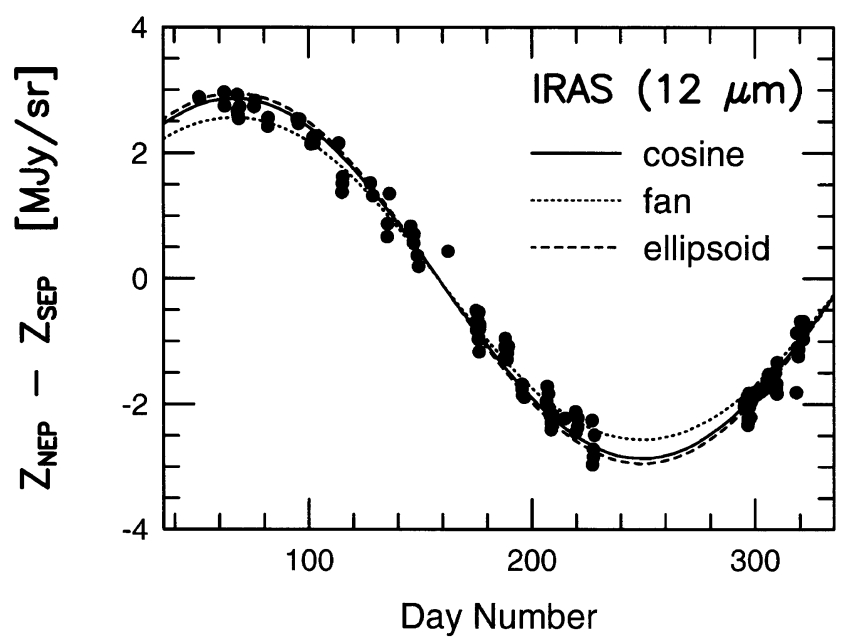

Fig. 6. Annual variation of the pole brightness difference at $12 \mu \mathrm{m}$ is fitted to the model calculation with $i=2 .^{\circ} 3$ and $\Omega=75^{\circ}$. The best fit line (solid one) is from the cosine model. The dashed line is from the ellipsoid model and dotted from the fan one.

good fits to the ZE data can be made by the ellipoid model, but best fits are done with the cosine model. This doesn't necessarily mean an existence of the central halo portrayed by the cosine model, because we haven't analysed inner parts of the zodiacal emission yet.

Acknowledgments. SSH was supported in part by the Korean Ministry of Education, Basic Science Research Institute grant No. BSRI97-5411, and SMK by the travel fund of Kangwon National Uni- versity, Republic of Korea.

\section{References}

Dermott, S. F., P. D. Nicholson, and B. A. Wolven, Preliminary analysis of the IRAS 1. Solar System dust data, in Asteroids, Comets, Meteors II, edited by C.-I. Lagerkvist, B. Lindblad, H. Lundstedt, and H. Rickman, $583 p p .$, HSC, Uppsala, 1986.

Dermott, S. F., K. Grogan, E. K. Holmes, and S. J. Kortenkamp, The dynamical structure of the zodiacal cloud, paper presented at ZCS workshop, Kobe, 1997.

Deul, E. R. and R. D. Wolstencroft, A physical model for thermal emission from the zodiacal dust cloud, Astron. Astrophys., 196, 277-286, 1988.

Giese, R. H. and C. V. Dziembowski, Suggested zodiacal light measurements from space probes, Planet. Space Sci., 17, 949-956, 1969.

Giese, R. H. and B. Kneißel, Three-dimensional models of the zodiacal cloud: II. Compatibility of proposed infrared models, Icarus, 81, 369$378,1989$.

Giese, R. H., B. Kneißel, and U. Rittich, Three-dimensional models of the zodiacal dust cloud: A comparative study, Icarus, 68, 395-411, 1986.

Hauser, M. G. and J. R. Houck, The zodiacal background in the IRAS data in Light on Dark Matter, edited by F. P. Israel, 39pp., Dordrecht, Reidel, 1986.

Hauser, M. G., T. N. Gautier, and F. J. Low, IRAS observations of the interplanetary dust emission, in Properties and Interactions of Interplanetary Dust, edited by R. Giese and P. Lamy, Reidel, Dordrecht, 43pp., 1985.

Hong, S. S. and S. M. Kwon, Connection between the infrared zodiacal emission and the visible zodiacal light, Vistas in Astronomy, 31, 11-21, 1988.

Hong, S. S. and S. M. Kwon, On the gegenschein and the symmetry plane, in Origin and Evolution of Interplanetary Dust, edited by A. C. LevasseurRegourd and H. Hasegawa, 147pp., Kluwer, Dordrecht, 1991

Hong, S. S. and I. K. Um, Inversion of the zodiacal infrared brightness integral, Astrophys. J., 320, 928-935, 1987.

Ishiguro, M., R. Nakamura, T. Watanabe, T. Mukai, H. Tanabe, I. Mann, H. Kimura, P. Hillebrand, and J. F. James, North-south asymmetry of the zodiacal light, in Proc. of the 29th ISAS Lunarand Planetary Symposium, edited by H. Mizutani, 64pp., ISAS, Kanagawa, 1996.

Kneißel, B. and I. Mann, Spatial distribution and orbital properties of zodiacal dust, in Origin and Evolution of Interplanetary Dust, edited by A. C. Levasseur-Regourd and H. Hasegawa, 139pp., Kluwer, Dordrecht, 1991.

Leinert, C., I. Richter, E. Pitz, and B. Planck, The zodiacal light from 1.0 to 0.3 A.U. as observed by the Helios space probes, Astron. Astrophys, 103, 177-188, 1981 .

Levasseur-Regourd, A. C. and R. Dumont, IRAS observations and local properties of interplanetary dust, Adv. Space Res., 10, (3)163-(3)170, 1990.

Misconi, N. Y., The symmetry plane of the zodiacal cloud near 1 AU, in Solid Particles in the Solar System, edited by I. Halliday and B. A. McIntosh, 49pp., Dordrecht, Reidel, 1980.

Murdock, T. L. and S. D. Price, Infrared measurements of the zodiacal light, Astron. J., 90, 375-386, 1985.

Reach, W. T., Zodiacal emission II. Dust near the ecliptic, Astrophys. J., 369, 529-543, 1991

Rittich, U., Die räumliche Verteilung des Interplanetaren Staubes: Modellrechnungen zur Interpretation von Zodiakallichtmessungen, Diploma Thesis, Ruhr University, Bochum, 1986.

Rowan-Robinson, M., J. Hughes, K. Vedi, and D. W. Walker, Modelling the IRAS zodiacal emission, MNRAS, 246, 273-278, 1990.

Temi, P., P. De Bernardis, S. Masi, G. Moreno, and A. Salama, Infrared emission from interplanetary dust, Astrophys. J., 337, 528-535, 1989.

Vrtilek, J. M. and M. G. Hauser, IRAS measurements of diffuse solar system radiation: Annual sky brightness variation and geometry of the interplanetary dust cloud, Astrophys. J., 455, 677-692, 1995.

S. M. Kwon (e-mail: smkwon@ceres.kangwon.ac.kr) and S. S. Hong (e-mail: sshong@astroism.snu.ac.kr) 